Madalena Sales Luis, Margarida Alcafache, Sara Ferreira, Ana Laura Fitas, Joana Simões Pereira, Íris Caramalho, Lurdes Lopes and Catarina Limbert*

\title{
Children with type 1 diabetes of early age at onset - immune and metabolic phenotypes
}

https://doi.org/10.1515/jpem-2019-0103

Received February 24, 2019; accepted May 27, 2019; previously published online July 6, 2019

\section{Abstract}

Objectives: We aimed to evaluate children with type 1 diabetes (T1D) with early age at onset (EAO) for clinical, immune and metabolic features in order to identify agerelated disease phenotypes.

Methods: Comparative study of two groups of T1D children: $\mathrm{EAO}$ ( $\leq 5$ years) and later age at onset (LAO; $>5$ years), regarding the presence of other autoimmune (AI) diseases, diabetes ketoacidosis and immunologic profile at onset and metabolic data 1 year after diagnosis. Statistical analysis was performed with significance set for $\mathrm{p}<0.05$. Results: The study included 137 children $(\mathrm{EAO}=52$, mean age $3.6 \pm 1.5$ [mean \pm standard deviation (SD)] and $\mathrm{LAO}=85$, mean age $10.4 \pm 2.9)$. EAO was more associated with concomitant AI diseases $(\mathrm{p}=0.032)$. Despite no differences in disease onset, EAO presented with lower C-peptide levels $(\mathrm{p}=0.01)$ and higher absolute lymphocyte number $(p<0.0001)$, with an inverse correlation between these two variables $(p=0.028)$. Additionally, the EAO group had a higher frequency of serum detection of three antibodies $(A b s)(p=0.0008)$, specifically insulin

*Corresponding author: Catarina Limbert, MD, PhD, Paediatric Endocrinology and Diabetology Unit, Hospital de Dona Estefânia, Centro Hospitalar de Lisboa Central, Rua Jacinta Marto, 1169-045 Lisbon, Portugal, Phone: 00351213596 540, Fax: 00351213126 667, E-mail: climbert@gmail.com

Madalena Sales Luis: Paediatric Endocrinology and Diabetology Unit, Hospital de Dona Estefânia, Centro Hospitalar de Lisboa Central, Lisbon, Portugal; and Paediatric Unit, Hospital São Francisco Xavier, Centro Hospitalar de Lisboa Ocidental, Lisbon, Portugal. https://orcid.org/0000-0002-0324-0486

Margarida Alcafache, Sara Ferreira, Ana Laura Fitas and Lurdes Lopes: Paediatric Endocrinology and Diabetology Unit, Hospital de Dona Estefânia, Centro Hospitalar de Lisboa Central, Lisbon, Portugal

Joana Simões Pereira: Paediatric Endocrinology and Diabetology Unit, Hospital de Dona Estefânia, Centro Hospitalar de Lisboa Central, Lisbon, Portugal; and Endocrinology Unit, Instituto Português de Oncologia, Lisbon, Portugal. https://orcid.org/00000002-7119-5058

Íris Caramalho: Gulbenkian Science Institute, Lisbon, Portugal
Abs $(\mathrm{p}=0.0001)$. One year after diagnosis, EAO had higher total daily insulin (TDI) dose $(\mathrm{p}=0.008)$, despite similar hemoglobin $\mathrm{A}_{1 \mathrm{c}}\left(\mathrm{HbA}_{1 \mathrm{c}}\right)$.

Conclusions: Our data show an association of EAO T1D with more AI diseases, higher number of Abs, lower initial insulin reservoir and higher insulin requirements 1 year after diagnosis. In this group, immune imbalance seems more evident and disease progression faster, probably reflecting distinct "immune environment" with different ages at disease onset. Further studies in the field of immunogenetics and immune tolerance are required, to improve patient stratification and find novel targets for therapeutic intervention.

Keywords: autoantibodies; $\beta$-cells; early onset; innate immunity; type 1 diabetes.

\section{Introduction}

Type 1 diabetes (T1D) is an autoimmune (AI) condition characterized by a selective and progressive destruction of pancreatic $\beta$-cells $[1,2]$. Children under $3-5$ years of age comprise a small proportion of all those with this disorder (less than $2 \%$ under 3 years of age) [3]. However, current evidence suggests a possible trend toward T1D diagnosis at a younger age [4]. Importantly, an annual increase of $5.4 \%$ in T1D before the age of 5 years is estimated, and it is predicted that this number will double in the coming years $[5,6]$

Although extensive scientific research has yielded important insights into the immune mechanisms involved in pancreatic $\beta$-cell destruction, little is known about the events that trigger the AI process and the factors dictating age at onset. In fact, few studies characterize early onset T1D, and it is not clear why $\beta$-cell destruction is so aggressive in younger children $[5,7]$.

T1D is characterized by the involvement of cellular immunity, genetic susceptibility linked to the human leukocyte antigen (HLA) system and about 50 other non-HLA loci, as well as the presence of specific circulating autoantibodies [8]. In addition to a strong genetic component, environmental factors are clearly involved 
in the etiology of T1D and may help in explaining its growing incidence [9].

Among potential environmental factors, some studies support a role for viral infections, particularly enteroviruses, as a trigger for the development of T1D [10].

These triggers can induce the innate immune system response and a cascade of events, including the expression of pro-inflammatory cytokines and chemokines with a strong Th1 and Th17 cell response, which allows for cytotoxic CD8 T activation [9, 11].

Other studies have directed attention to a population of regulatory $\mathrm{T}$ cells (Tregs) which play a crucial role in the maintenance of homeostasis and self-tolerance of the immune system $[5,12,13]$.

Furthermore, cell autonomous responses and possible differences in the early education of the immune system should be considered as factors that might affect the degree of $\beta$-cell damage and the transition from innate immunity to adaptive immunity [10].

The appearance of pancreatic autoantibodies in the serum is the first sign of the initiation of the $\beta$-cell-specific AI process and characterizes the stage 1 of T1D. It reflects a critical increase in the risk of progression to diabetes, which is faster when the seroconversion with multiple autoantibodies occurs before the age of 3 years [14-16].

Children with T1D with early age at onset (EAO) pose a series of important challenges to health care professionals. The comprehension of the mechanisms dictating age at T1D onset may contribute to identify different phenotypes of the disease and potentially to disclose new individualized therapies.

In this study, we aimed to evaluate children with T1D before and after 5 years of age in terms of AI background, number and type of pancreatic autoantibodies, disease severity at onset and its correlation with white blood count, and C-peptide levels and metabolic control 1 year after the diagnosis.

\section{Materials and methods}

\section{Study design}

We performed a retrospective study including all children and adolescents (up to 18 years of age) diagnosed with T1D in a central pediatric hospital, in the period between January 2008 and March 2017. All cases of neonatal diabetes (onset $\leq 6$ months) were excluded. The sample was divided into two groups according to age at disease onset: $\mathrm{EAO} \leq 5$ years and later age at onset $(\mathrm{LAO})>5$ years.

Informed consent was obtained from all individuals included in this study.
The research related to human use complied with all the relevant national regulations, institutional policies and was in accordance with the tenets of the Helsinki Declaration, and has been approved by the authors' Institutional Review Board.

\section{Data collection}

Data was collected from records of each patient and included: demographic data, presence of other AI diseases until the end of the study (March 2018) and T1D presentation (diabetic ketoacidosis [DKA] or non-ketoacidosis). A peripheral blood sample was collected, within the first 3 days after diagnosis, for the following parameters: complete white blood count (WBC), fasting C-peptide levels and pancreatic autoantibodies (glutamic acid decarboxylase 65 autoantibodies [GAD], tyrosine phosphatase-like insulinoma antigen 2 [IA2] and insulin autoantibodies [IAA]).

Screening for other AI diseases (celiac disease and thyroiditis) was performed at the time of diagnosis and annually.

One year after diagnosis, total daily insulin (TDI) (units per kg of body weight $[\mathrm{U} / \mathrm{kg}])$ and hemoglobin $\mathrm{A}_{1 \mathrm{c}}\left(\mathrm{HbA}_{1 \mathrm{c}}, \%\right)$ were evaluated.

Fasting C-peptide was determined by chemiluminescence (Immulite 2000 Siemens, Erlangen, Germany), pancreatic autoantibodies were determined by radioimmunoassay (Medipan $\mathrm{GmbH}$, Hamburg, Germany), and glycated hemoglobin $\left(\mathrm{HbA}_{1 \mathrm{c}}\right)$ by chromatography (Variance 2-Biorad, Hercules, CA, USA).

DKA was defined as occasional blood glucose concentration $>200 \mathrm{mg} / \mathrm{dL}$ ( $>11 \mathrm{mmol} / \mathrm{L}$ ), venous $\mathrm{pH}<7.3$ or bicarbonate $<15 \mathrm{mmol} / \mathrm{L}$ and positive ketonemia or ketonuria. Severity of disease at onset was defined by the presence or absence of DKA at diagnosis.

\section{Statistical analysis}

Statistical analysis was performed using GraphPad Prism ${ }^{\circledast}$ (GraphPad Software, La Jolla, CA, USA) and Microsoft office Excel $365^{\circledR}$ (Microsoft, Redmond, WA, USA). Data normally distributed is presented as mean \pm standard deviation (SD) or as median and interquartile range (25th-75th percentile). Chi-square $\left(\chi^{2}\right)$ tests were used for statistical comparisons for dichotomous and categorical variables. The MannWhitney t-test was applied for unequally distributed variables. A p-value less than 0.05 was considered significant.

\section{Results}

\section{Characteristics of the study population}

The clinical characteristics of the 137 children who met the inclusion criteria are described in Table 1. Fifty-two children were included in the EAO group (mean age $3.6 \pm 1.5$ years; $56 \%$ males) and 85 children were included in the LAO group (mean age $10.4 \pm 2.9$ years; $56 \%$ males).

The mean duration of diabetes was $4.1 \pm 2$ years in the EAO group and $3.8 \pm 2$ years in the LAO group. 
Table 1: Clinical characteristics of the study population.

\begin{tabular}{lrr}
\hline & $\leq 5$ years & p-Value \\
\hline $\mathrm{n}, \%$ & $52(38 \%)$ & $85(62 \%)$ \\
Age, years (mean \pm SD) & $3.6 \pm 1.5$ & $10.4 \pm 2.9$ \\
Gender (male/female) & $29 / 23$ & $48 / 37$ \\
Diabetes duration, years (mean \pm SD) & $4.1 \pm 2$ & $3.8 \pm 2$ \\
Insulin treatment (MDI/CSII) & $37 / 15$ & $85 / 0$ \\
Al background (yes/no) & $9 / 43$ & $5 / 80$ \\
Diabetes presentation (DKA/non-DKA) & $15 / 30$ & $35 / 49$ \\
TDI, U/kg 1 year after diagnosis (median [25th-75th percentile]) & $0.72(0.57-0.87)$ & $0.6(0.49-0.79)$ \\
\hline
\end{tabular}

Al, autoimmune; CSII, continuous subcutaneous insulin infusion; DKA, diabetic ketoacidosis; MDI, multiple daily injections; SD, standard deviation; TDI, total daily insulin.

At the end of the first year of therapy, 73 children from the EAO group were treated with multiple daily injections (MDIs) and 15 started on continuous subcutaneous insulin infusion (CSII), whilst the LAO group were all on MDI.

\section{EAO was more associated with other autoimmune diseases}

In the EAO group, nine children presented another $\mathrm{AI}$ disease compared to five children in the LAO group $(p=0.032)$ (Table 1) until the end of the study. In the EAO group, five patients had AI thyroiditis and four had celiac disease. One child was diagnosed with celiac disease 1 year before T1D and three had a diagnosis of another AI disease at the time of diabetes onset. In all the others, the associated AI disease was diagnosed 2-4 years after T1D. In the LAO group, two patients had celiac disease diagnosed at the time of diabetes onset and 1 year after, two had AI thyroiditis diagnosed 1 to 3 years after diabetes onset, and one child had juvenile idiopathic arthritis diagnosed 6 years before T1D.

\section{Innate immune response is significantly different in children $\leq 5$ years and is related to residual $\beta$-cell function}

The EAO group had higher initial absolute lymphocyte counts (median $4.5 \times 10^{3}$ cells/L [3.2-5.7] vs. $3.1 \times 10^{3}$ cells/L [2.4-3.7], $\mathrm{p}<0.0001)$ and lower neutrophil numbers (median $2.9 \times 10^{3}$ cells/L [2.4-3.7] vs. $3.5 \times 10^{3}$ cells/L [2.4-4.9], $\mathrm{p}=0.39$ ) (Figure 1). Children in the EAO group also had lower fasting C-peptide levels at disease onset (median $0.2 \mathrm{ng} / \mathrm{mL}$ [0.1-0.3] vs. $0.3 \mathrm{ng} / \mathrm{mL}$ [0.2-0.5], $\mathrm{p}=0.01)$.
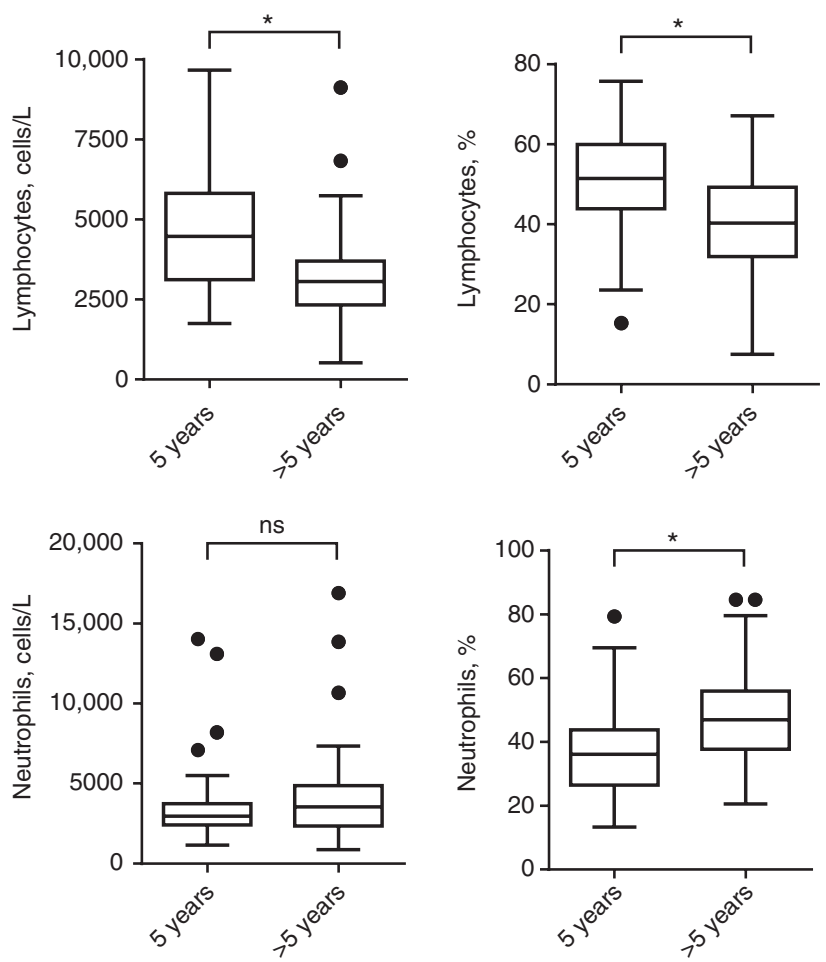

Figure 1: Lymphocytes and neutrophils at diagnosis in each of the two groups.

Percentages and absolute counts (cells/L) of lymphocytes and neutrophils from a peripheral blood sample collected at diabetes onset (within 3 days). Boxes show median (with $25 \%$ and $75 \%$ quartiles). Whiskers and outliers were plotted using the Tukey method. Ns, non-statistical. ${ }^{*} \mathrm{p}<0.05$ by the Mann-Whitney test.

Considering patients from both groups, we found an association between C-peptide levels $\leq 0.4 \mathrm{ng} / \mathrm{mL}$ and higher lymphocyte count at T1D onset $(p=0.0286)$ (Figure 2A). Moreover, there seems to be a negative correlation between fasting C-peptide levels and absolute lymphocyte count $(r=-0.215 ; \mathrm{p}=0.008)$ (Figure 2B). 
A
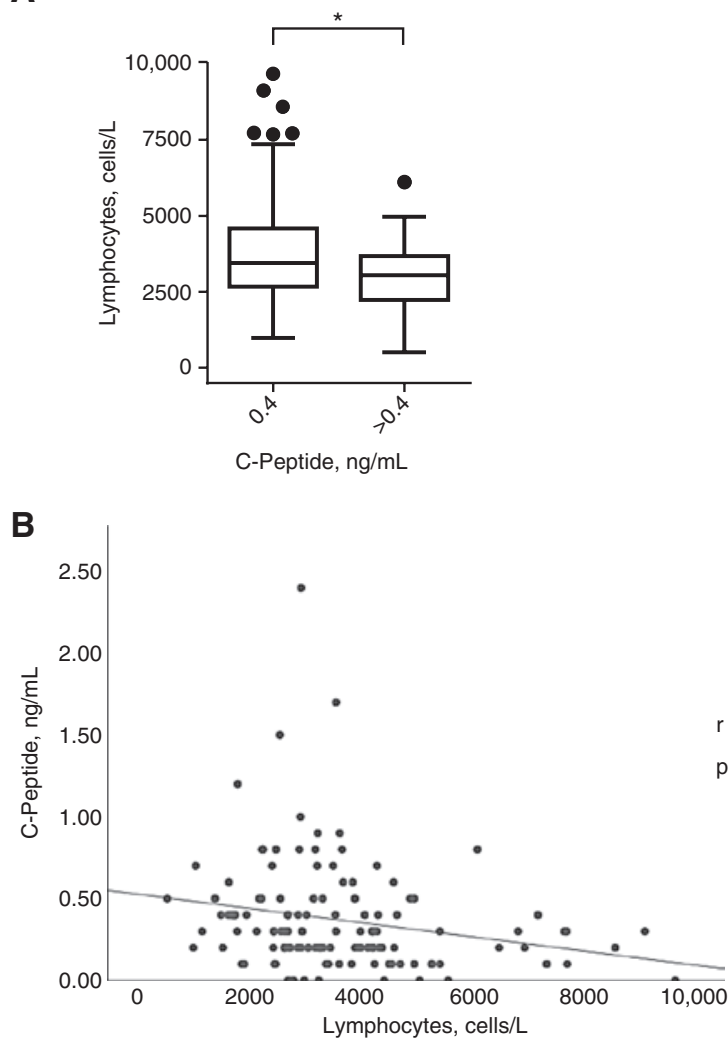

Figure 2: Correlation between fasting C-peptide levels and absolute lymphocyte counts.

Lymphocyte count (cells/L) and C-peptide levels $(\mathrm{ng} / \mathrm{mL}$ ) from peripheral blood at diabetes onset (within 3 days). All patients were included from both groups. (A) A cut-off value for C-peptide levels $\leq 0.4 \mathrm{ng} / \mathrm{mL}$ and $>0.4 \mathrm{ng} / \mathrm{mL}$ was considered. Box shows median (with $25 \%$ and $75 \%$ quartiles). Whiskers and outliers were plotted using the Tukey method. ${ }^{*} \mathrm{p}<0.05$ by the Mann-Whitney test. (B) Absolute lymphocyte counts are shown in correlation to fasting C-peptide levels. $\mathrm{p}<0.05$ by Spearman's rank order correlation.

\section{EAO had higher number of positive autoantibodies at presentation}

At the time of diagnosis, the EAO group had more children with three positive autoantibodies than the LAO group $(\mathrm{p}=0.008)$ (Figure 3). Overall, 10 children $(7.3 \%)$ had all antibodies (Abs) negative. Regarding the type of $\mathrm{Ab}$, the EAO group had more IAA (30 vs. $22, p=0.0001$ ) than LAO and less proportion of anti-GAD ( $35 \mathrm{vs.} 61, \mathrm{p}=0.85$ ) and antiIA2 (34 vs. $60, p=0.77$ ), although the difference in these last two autoantibodies was not statistically significant.

\section{EAO and LAO had similar disease presentation}

Evaluation of T1D severity at onset revealed that the proportion of patients presenting with ketoacidosis or

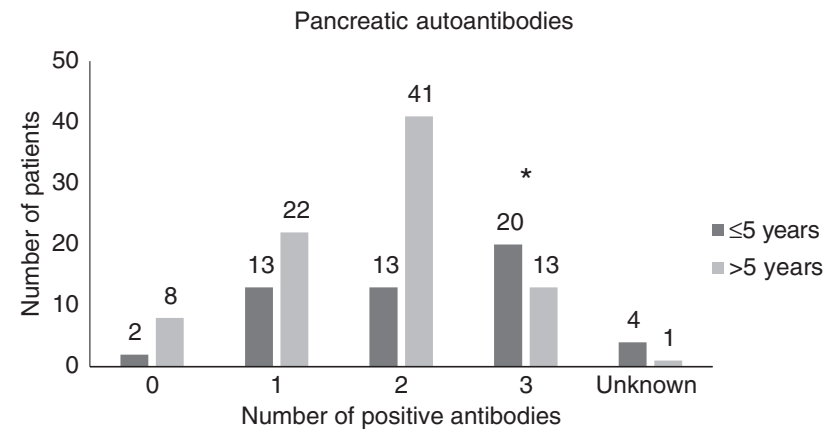

Figure 3: Pancreatic autoantibodies.

Number of children with $0,1,2$ or 3 positive pancreatic autoantibodies according to age at disease onset. ${ }^{*} p<0.05$ by the chi-square test. Autoantibodies performed: glutamic acid decarboxylase 65 (GAD), tyrosine phosphatase-like insulinoma antigen 2 (IA2) and insulin autoantibodies (IAA).

non-ketoacidosis was similar in both groups (15/30 vs. $35 / 49, p=0.24)$ (Table 1). In eight patients, data for the presentation were not available.

\section{One year after diagnosis, children with EAO require significantly higher insulin levels}

One year after disease onset, $\mathrm{HbA}_{1 \mathrm{c}}$ levels were similar in both groups $(8.5 \pm 0.9 \%$ vs. $8.4 \pm 1.4 \%, p=0.11)$. Notwithstanding, EAO required higher doses of insulin when compared to the LAO group (median $0.72 \mathrm{U} / \mathrm{kg}$ [0.57-0.87] vs. $0.6 \mathrm{U} / \mathrm{kg}$ [0.49-0.79], $\mathrm{p}=0.008$ ) (Table 1). The TDI of children with CSII did not influence the differences between the two groups.

\section{Discussion}

The association between T1D and other AI diseases, such as Hashimoto's thyroiditis and celiac disease, is well known and is likely related to a common genetic susceptibility $[17,18]$. In fact, a high proportion of children and adolescents with T1D have detectable islet autoantibodies, as well as other organ-specific autoantibodies [19, 20]. In these patients, the incidence of thyroiditis is approximately $3-8 \%$ and the prevalence of celiac disease ranges from 1 to $10 \%$ [20]. One of the main risk factors for the development of another AI disease is the duration of T1D [20]. Our data show an association of EAO with a higher frequency of other AI diseases. Importantly, our results also show that $44 \%$ of EAO patients had another AI disease previously or at the time of T1D diagnosis, indicating that T1D 
duration is not the only factor underlying increased risk to develop additional AI disease. However, larger multicentric studies are required to support these results, together with HLA typing.

T cell-mediated $\beta$-cell destruction is the predominant effector mechanism in T1D, but is not its primary cause. The mechanism behind the activation of the immune system and the cellular auto-attack is still not fully clear [21]. Our study shows that in EAO, the immune response seems significantly different.

EAO patients present at diagnosis lower neutrophil counts, higher lymphocyte numbers and less residual $\beta$-cell function, as reflected by lower C-peptide levels. Notably, we found an inverse correlation between lymphocyte number and fasting C-peptide levels. These results suggest that in the EAO group, exacerbated immune response has an important role in the anticipation of $\beta$-cell lesion.

To the best of our knowledge, few studies focused on this young group of patients until now. Szypwoska et al. studied the role of Tregs in the immune process in children under 5 years of age. They reported that younger children had low levels of C-peptide and a low number of FoxP3 Tregs as well as immunosuppressive cytokines, such as interleukin-10 (IL-10) and transforming growth factor- $\beta$ (TGF- $\beta$ ) [5]. It is known that abnormalities in Tregs may disrupt the balance between activation and suppression of the immune system leading to $\beta$-cell destruction. However, innate immune cells were not assessed in this study [22, 23].

Leete et al. found that the extension and progression of $\beta$-cell loss varies with age, and patients who develop T1D early in life (before the age of 7 years) tend to lose a superior extension of $\beta$-cells than those diagnosed in adolescence or later in life [1]. They suggest that insulitis occurs in two distinct profiles according to the age at onset, with the main difference being the proportion of $\mathrm{CD} 2 \mathrm{O}^{+} \beta$-cells present within the infiltrate, which leads to distinct aggressive phenotypes. Moreover, the proportion of insulin-containing islets is much lower in patients diagnosed at an early age which is consistent with our study and previous studies [1, 24].

Recently, Cabrera et al. reinforced the hypothesis of subgroups with different immune response and disease progression. They used an index to calculate the ratio between inflammatory and regulatory genes and found that patients having lower innate inflammatory bias at the time of clinical onset were more likely to have slower rates of decline in residual insulin secretion. However, in this study, findings were independent from the age at clinical onset [25].
Despite the more evident immune imbalance in younger children, our data show that it was not associated with a more severe presentation, as both groups had similar number of DKA episodes. We believe that this similar presentation is related to the fact that symptoms are more visible at younger ages. Also, caregivers of younger children are more alert to symptoms and they probably seek medical advice sooner.

In our cohort, EAO patients had significantly more IAA. Other studies have similar results [26-29]. Kimpimäki et al. showed that IAAs are usually the first islet autoantibodies to appear in the natural history of T1D and they are influential identifiers of disease progression in children [26]. Higher IAA titer at younger ages of onset is consistent with the concept that these patients develop a more aggressive disease course as IAAs appear to be early markers of $\beta$-cell destruction [26-28]. In agreement, children with late-onset autoimmunity are less likely to present with IAA at seroconversion [29].

Individuals with early-onset islet autoimmunity present more frequently with multiple autoantibodies at the first positive sample. This may be due to the presence of multiple autoantibodies at seroconversion or otherwise be a consequence of a shorter progression period from single to multiple autoantibodies, such that the first sample is more likely to miss the short period of single autoantibody positivity [29].

This is consistent with our results, as we have a substantially higher number of patients with three positive autoantibodies in the EAO group at the time of diagnosis.

Knip et al. studied the role of humoral $\beta$-cell autoimmunity in T1D and they stated that the first signs of $\beta$-cellspecific autoimmunity may appear early during the first months of life [30]. Hence it is likely that T1D appearance in young children reflects a process that began during the first 2 years of life or even during pregnancy [14]. In agreement, most of the children diagnosed with T1D before puberty seroconvert to autoantibody positivity before the age of 3 years $[14,30]$.

On the other hand, in our study, 10 children (7.3\%) presented with no autoantibody positivity. This proportion is similar to the frequency described in other studies ranging from 3.6 to $7.9 \%$ [30]. These patients should nevertheless be tested for other islet autoantibodies, namely ZnT8, which are currently not performed in our center.

Regarding the follow-up 1 year after diagnosis, our results demonstrate that the EAO group requires significantly higher doses of insulin. Abdul-Rasoul et al. in a study with diabetic children younger than 12 years showed that children below 5 years had lower remission rates than the older group [31]. Another study also demonstrated 
that in children under the age of 5 years, partial remission is unusual [32]. This reflects the more aggressive and rapid destruction of $\beta$-cells and thus less residual insulin at the time of diagnosis [31].

Despite these results, the metabolic control between the two groups was similar. This is probably related to more careful supervision by caregivers in younger children.

In conclusion, our data show an association of EAO with more AI diseases, lower initial insulin reservoir (although similar disease presentation) and higher insulin requirements 1 year after diagnosis to achieve similar metabolic control. In EAO children, immune imbalance is more evident and disease progression is more rapid. Decreased neutrophil blood counts at diagnosis suggest the involvement of the innate immune system in pancreatic $\beta$-cell lesion. Pancreas donor studies would help to better understand the immunological peripheral blood changes.

Our results reveal important differences in the "immune environment" of children with different ages at onset, which may reflect more aggressive triggers of disease or "age-related" immune-genetic susceptibility phenotypes.

We believe that EAO T1D represents a suitable disease model to understand distinct T1D phenotypes. Further studies in the field of immunogenetics and immune tolerance are required to improve patient stratification and find novel targets for therapeutic intervention.

Author contributions: MSL was responsible for data collection and wrote the manuscript. MA collected the data, performed statistical analysis and revised the manuscript. SF collected the data and revised the manuscript. ALF was enrolled in patient recruitment and revised the manuscript. JSP initiated data collection and revised the manuscript. IC and LL revised the manuscript. CL initiated and supervised the project, co-wrote the manuscript and is the guarantor of this work. All authors discussed the results and approved the final version of the manuscript. Conflict of interest: Nothing to declare.

Research funding: Nothing to declare.

Employment or leadership: Nothing to declare.

Honorarium: Nothing to declare.

\section{References}

1. Leete P, Willcox A, Krogvold L, Dahl-øørgensen K, Foulis AK, et al. Differential insulitic profiles determine the extent of b-cell destruction and the age at onset of type 1 diabetes. Diabetes 2016;65:1362-9.
2. Klinke DJ. Extent of beta cell destruction is important but insufficient to predict the onset of type 1 diabetes mellitus. PLoS One 2008;3:1374.

3. Daneman D, Frank M, Perlman K, Wittenberg J. The infant and toddler with diabetes: challenges of diagnosis and management. Paediatr Child Health 1999;4:57-63.

4. Patterson CC, Dahlquist GG, Gyürüs E, Green A, Soltész G, et al. Incidence trends for childhood type 1 diabetes in Europe during 1989-2003 and predicted new cases 2005-20: a multicenter prospective registration study. Lancet 2009;373:2027-33.

5. Szypowska A, Stelmaszczyk-Emmel A, Demkow U, Łuczynski W. Low frequency of regulatory $T$ cells in the peripheral blood of children with type 1 diabetes diagnosed under the age of five. Arch Immunol Ther Exp 2012;60:307-13.

6. Cody Declan. Infant and toddler diabetes. Arch Dis Child 2007;92:716-9.

7. Poudel A, Savari O, Striegel D, Periwal V, Taxy J, et al. Beta-cell destruction and preservation in childhood and adult onset type 1 diabetes. Endocrine 2015;49:693-702.

8. Boudiaf L, Bouziane D, Smara M, Meddour Y, Haffaf EM, et al. Could ZnT8 antibodies replace ICA, GAD, IA2 and insulin antibodies in the diagnosis of type 1 diabetes? Curr Res Transl Med 2018;66:1-7.

9. Waught K, Snell-Bergeon J, Michels A, Dong F, Steck AK, et al. Increased inflammation is associated with islet autoimmunity and type 1 diabetes in the Diabetes Autoimmunity Study in the Young (DAISY). PLoS One 2017;12:0174840.

10. Beeck AO, Eizirik D. Viral infections in type 1 diabetes mellitus - why the $\beta$ cells? Nat Rev Endocrinol 2016;12:263-73.

11. Walker LS, von Herrath M. CD4 T cell differentiation in type 1 diabetes. Clin Exp Immunol 2016;183:16-29.

12. Visperas A, Vignali D. Are Tregs defective in type 1 diabetes and can we fix them? J Immunol 2016;197:3762-70.

13. Ludvigsson J. Therapies to preserve b-cell function in type 1 diabetes. Drugs 2016;76:169-85.

14. Parikka V, Näntö-Salonen K, Saarinen M, Simell T, Ilonen J, et al. Early seroconversion and rapidly increasing autoantibody concentrations predict prepubertal manifestation of type 1 diabetes in children at genetic risk. Diabetologia 2012;55:1926-36.

15. Insel RA, Dunne JL, Atkinson MA, Chiang JL, Dabelea D, et al. Staging presymptomatic type 1 diabetes: a scientific statement of JDRF, the Endocrine Society, and the American Diabetes Association. Diabetes Care 2015;38:1964-74.

16. Couper JJ, Haller MJ, Greenbaum CJ, Ziegler AG, Wherrett DK, et al. ISPAD Clinical Practice Consensus Guidelines 2018: stages of type 1 diabetes in children and adolescents. Pediatr Diabet 2018;(Suppl 19):20-7.

17. Fröhlich-Reiterer EE, Hofer S, Kaspers S, Herbst A, Kordonouri O, et al. Screening frequency for celiac disease and autoimmune thyroiditis in children and adolescents with type 1 diabetes mellitus - data from a German/Austrian multicentre survey. Pediatr Diabetes 2008;9:546-53.

18. Krzewska A, Ben-Skowronek I. Effect of associated autoimmune diseases on type 1 diabetes mellitus incidence and metabolic control in children and adolescents. Biomed Res Int 2016;2016:6219730.

19. Barker J. Clinical review: type 1 diabetes-associated autoimmunity: natural history, genetic associations, and screening. J Clin Endocrinol Metab 2006;91:1210-7. 
20. Mahmud FH, Elbarbaryd NS, Frohlich-Reitererc E, Holl RW, Kordonouri O, et al. ISPAD Clinical Practice Consensus Guidelines 2018: other complications and associated conditions in children and adolescents with type 1 diabetes. Pediatr Diabetes 2018;(Suppl 27):275-86.

21. Bougnères $P$, Valleron $A J$. Causes of early-onset type 1 diabetes: toward data-driven environmental approaches. J Exp Med 2008;205:2953-7.

22. Graham K, Sutherland R, Mannering SI, Zhao Y, Chee J, et al. Pathogenic mechanisms in type 1 diabetes: the islet is both target and driver of disease. Rev Diabet Stud 2012;9:148-68.

23. Vrabelova Z, Hrotekova Z, Hladikova Z, Bohmova K, Stechova K, et al. $\mathrm{CD} 127^{-}$and $\mathrm{FoxP3}^{+}$expression on $\mathrm{CD}_{25} 5^{+} \mathrm{CD} 4^{+} \mathrm{T}$ regulatory cells upon specific diabetogeneic stimulation in high-risk relatives of type 1 diabetes mellitus patients. Scand J Immunol 2008;67:404-10.

24. Arif S, Leete P, Nguyen V, Marks K, Nor NM, et al. Blood and islet phenotypes indicate immunological heterogeneity in type 1 diabetes. Diabetes 2014;63:3835-45.

25. Cabrera SM, Engle S, Kaldunski M, Jia S, Geoffrey R, et al. Innate immune activity as a predictor of persistent insulin secretion and association with responsiveness to CTLA4-Ig treatment in recent-onset type 1 diabetes. Diabetologia 2018;61:2356-70.
26. Kimpimäki T, Kulmala P, Savola K, Kupila A, Korhonen S, et al. Natural history of $\beta$-cell autoimmunity in young children with increased genetic susceptibility to type 1 diabetes recruited from the general population. Clin Endocrinol Metab 2002;87:4572-9.

27. Bizzarri C, Benevento D, Ciampalini P, Ippolita P. Clinical presentation and autoimmune characteristics of very young children at the onset of type 1 diabetes mellitus. J Pediatr Endocrinol Metab 2010;23:1151-7.

28. Pietropaolo M, Towns R, Eisenbarth GS. Humoral autoimmunity in type 1 diabetes: prediction, significance, and detection of distinct disease subtypes. Cold Spring Harb Perspect Med 2012;2:012831.

29. Frohnert BI, Ide L, Dong F, Barón AE, Steck AK, et al. Late-onset islet autoimmunity in childhood: the Diabetes Autoimmunity Study in the Young (DAISY). Diabetologia 2017;60:998-1006.

30. Knip M, Siljander H, Ilonen J, Simell O, Veijola R. Role of humoral beta-cell autoimmunity in type 1 diabetes. Pediatr Diabetes 2016; Suppl 22:17-24.

31. Abdul-Rasoul M, Habib H, Al-Khouly M. 'The honeymoon phase' in children with type 1 diabetes mellitus: frequency, duration, and influential factors. Pediatr Diabetes 2006;7:101-7.

32. Muhammad BJ, Swift PG, Raymond NT, Botha JL. Partial remission phase of diabetes in children younger than age 10 years. Arch Dis Child 1999;80:367-9. 